\title{
Rapid Diagnostic Test Cassette: A Source of DNA for Malaria Diagnosis by PCR and a Quality Control Tool for the Test Performance
}

\author{
Godwill A. Engwa ${ }^{12}$ \\ Olivia A. Achundoh ${ }^{1}$ \\ Wilfred F. Mbacham ${ }^{1}$ \\ ${ }^{1}$ Laboratory for Public Health Research Biotechnologies (LAPHER-Biotech), \\ The Biotechnology Center, University of Yaounde I, Yaounde, Cameroon \\ ${ }^{2}$ Department of Biotechnology, International Bio-Research Institute \\ Ugwogo-Nike, Enugu State Nigeria
}

\section{Doi:10.5901/ajis.2013.v2n13p69}

Abstract

RDT is a malaria diagnostic tool used for early diagnosis of the disease. Though reliable, their challenging performance demands for continuous quality control monitoring. In this study, we evaluated the use of RDT cassette as a source of DNA to detect malaria parasite by PCR and in quality control of RDT in malaria diagnosis. Malaria parasite DNA was extracted from both immediately used ("fresh") and archived RDT cassettes and was amplified by PCR for msp 1, msp 2 and glurp gene and the test performance of RDT was evaluated comparing PCR results for msp 2 with those of microscopy and RDT. Findings from this study showed RDT as a source of malaria parasite DNA as DNA was successful extracted from RDT cassettes and amplified by PCR. Comparing $P C R$, microscopy and $R D T$ results showed $P C R$ to be most reliable in detecting malaria parasite. It was confirmed that RDT cassette is a potential cost effective source of DNA in molecular epidemiology studies to detect malaria parasite by PCR and in quality control of malaria diagnostic test performance.

Keywords: RDT, PCR, malaria parasite, DNA, microscopy, msp 2

\section{Introduction}

Malaria remains the most deadly tropical disease in the world, responsible for about 225 thousand clinical cases and 1 million deaths yearly. The greatest disease burden lies within the sub-Saharan region of Africa particularly affecting children and pregnant women (WHO, 2010).

In recent time, there is so much concern in the control of the disease among which are the use of Artemisinin based combination therapy(ACT), long lasting insecticide treated nets, Intermittent preventive treatments and case management of the disease (WHO, 2010). Case management defined by rapid diagnosis and prompt treatment is a control strategy of interest to minimize complications as well as reduce mortality. Rapid Diagnostic Test (RDT), which permits malaria diagnosis within 15-30 minutes, can be used at community level for case management (Tangpukdee et al, 2009; Chansuda et al, 2007; Moody, 2002). Though reliable, RDTs are limited in detecting sub-microscopic parasitaemia levels.

The World Health Organization (WHO) recommendations for performing quality assurance of RDTs among other things demands that quality control (QC) of RDT should be monitored in the field by comparing the results of RDTs with those of microscopy monthly (McMorrow et al, 2010; 2008; WHO, 2008; NVBCP, 2007). Though microscopy remains the goal standard for diagnosis, it 
has been challenging using microscopy as reference tool for QC due to difficulties to re-read slides usually covered with fungi growth as a result of poor conservation facilities in local health centers. alternatively, dried blood on geimsa stained slides could be collected and used as a source of DNA for PCR amplification to detect malaria parasite but the harsh staining of blood with geimsa may hampers the DNA extraction process and PCR amplification (Cnops et al, 2010; Scopel et al, 2004; Edoh et al, 1997)..

RDT is an immunochromatographic technique which contains fixed antibodies bound on nitrocellulose strips to detect soluble antigens of the parasite in blood to produce visible bands (Tangpukdee et al, 2009; Chansuda et al, 2007; Moody, 2002). Previous studies have shown RDT as a source of DNA for molecular analyses by PCR in the detection of malaria parasite (Cnops et al, 2011 Ishengoma et al, 2011, Veron and Carme, 2006). In this study, we investigated the use of Rapid Diagnostic Test cassettes as a source of DNA to detect malaria parasite by PCR and in quality control of RDT in malaria diagnosis.

\section{Methods}

\subsection{Study Site}

This study was conducted at the laboratory for Public Health Research Biotechnology (LAPHERBiotech) at the Biotechnology Centre (Nkolbisson) of the University of Yaounde I, Yaounde, Cameroon.

\subsection{Study Procedure}

This study was part of the Research on the economics of ACTs (REACT) project in Cameroon. A malaria positive blood sample was obtained from the etoug-ebe Baptist Health Centre, Yaounde in 2010. About 5ul of the blood sample was dispensed unto the sample well followed by 4 drops of assay diluents into the diluent well of 4 SD Bioline test kit for $P$. falciparum and pan malaria antigens according to the manufacturers' instructions. The RDT cassettes were allowed for 15-20 minutes and result were read. DNA was extracted from the 4 immediately used ("fresh") RDT cassettes and amplified by PCR for merozoite surface protein $1(\mathrm{msp} 1)$, merozoite surface protein (msp 2), and glutamate rich protein (glurp) genes of Plasmodium falciparum. DNA was then extracted from forty five 18 months old archived RDTs collected in 2009 and amplified by PCR for msp 2 gene to detect malaria parasite. As part of QC testing of RDTs, PCR results were compared with those of RDT and microscopy previously screened for malaria.

\subsection{DNA extraction, PCR amplification and electrophoresis}

The RDT cassettes (previously screened for malaria) were opened and the nitrocellulose membrane taken off. The portion containing the sample blotting side of the nitrocellulose membrane was carefully excised using sterile scissor and forcep and transferred in $1.5 \mu l$ eppendorf tubes. DNA was extracted following the chelex boiling method as described by Plowe et al, (1995).

Amplification of msp 2, msp 1 and glurp genes was done by nested PCR using the T3 thermal cycler machine (Biometra) as described by Mbacham et al, (2008) using primers from Malaria Research and Reference Reagent Resource Center (MR4).

$1.5 \%$ agarose gel (Seakem Nusieve) was prepared and was visualized under UV transilluminator (High Performance) and photographs taken using a digital camera.

\subsection{Data and Statistical analysis}

From the electrophoresis result, distance migrated by DNA was plotted against log of DNA 
molecular weight marker to determine the molecular sizes of PCR products. The genetic diversity was calculated as the number of alleles present divided by the total number of distinct DNA fragments. Complexity of infection was defined as the average number of alleles present per individual was calculated as the total number of distinct DNA fragments divided by the total number of samples.

SPSS 16 statistical software was used for data analysis and graph plotting and confidence interval was taken at $95 \%$.

\section{Results}

\subsection{PCR, Microscopy and RDT}

DNA was extracted from both "fresh" and archived RDT cassettes and successfully amplified by PCR (Figure 1). In total 41 out of 47 RDT samples were positive by PCR. Among these samples, 4 from "fresh" RDT cassettes were positive by PCR for msp 2, msp 1 and glurp genes and 37 from archived RDT cassettes was positive for msp 2 gene.

Comparing the PCR results with those of RDT and microscopy showed that all 4 "Fresh" RDT cassettes positive for malaria were all positive for malaria parasite by PCR. Among the 37 archived RDT samples positive by PCR, 12 were RDT and microscopy positive samples, 14 were RDT negative but microscopy positive samples and 11 were RDT positive but microscopy negative samples. From this results, PCR was more performant in malaria diagnosis as it could detect malaria parasite in both RDT and microscopy negative sample (RDT and microscopy false negatives). Results are shown on table 1.

\subsection{Genetic diversity of Plasmodium falciparum}

Genotyping the 37 msp 2 PCR positive samples from archive RDT cassettes, genetic diversity of $P$. falciparum was $35.4 \%$ (17 alleles of 48 fragments) and complexity of infection was 1.3 (48 DNA fragments for 37 samples) (Table 2). The infection types were single or mixed with a dominance of single genotype in 28 samples (75.7\%). Mixed infection was $24.3 \%$ ( 9 of 37) existing as 6 double $(16.2 \%)$ and 3 triple (8.1\%) mixed infections respectively (Figure 2$) .17$ alleles were detected with band sizes ranging from $400 \mathrm{bp}-630 \mathrm{bp}$. The most dominant allele was the 500bp with a frequency of $38.3 \%$ (Figure 3 ).

\section{Discussion}

The introduction of RDT in malaria diagnosis has greatly improved on the case management of the disease and its control. Though this diagnostic tool is effective in early diagnosis of the disease, their test performance remains a major worry. This has edge WHO to recommend QC of RDT by monitoring their test performance using microscopy for at least 20 malaria positive and negative RDT samples (McMorrow et al, 2010; 2008; WHO, 2008; NVBCP, 2007). Microscopy remains the most effective tool for malaria diagnosis and used as the gold standard but their use in quality control remains challenging because it necessitates that control slides needs to be prepared alongside certain test RDTs (Igbinosa et al, 2010). Also, re-reads of the slides in a long run could produce false negative or positive results due to poor long term storage of the slides enhancing the development of fungi growth (Ishengoma et al, 2011).

Recently, RDT was shown to serve as a source of DNA to detect malaria parasite by PCR (Cnops et al, 2011 Ishengoma et al, 2011, Veron and Carme, 2006). In these studies, malaria parasite was detected in 18 months old archive RDT cassettes which were stored at room temperature. This suggests that PCR on "RDT" as a source of DNA could be used for QC to monitor the performance of RDT and will not be affected by the storage condition. From the results 
obtained in this study, we confirm that DNA could successfully be extracted and amplified by PCR from both "fresh" and archived RDT cassettes. Comparing the results of PCR with RDT, and microscopy, PCR showed to be more reliable in detecting malaria parasite as PCR could detect malaria parasite in RDT and microscopy negative samples. Similar studies using RDT as a source of DNA showed PCR to be more effective than microscopy or RDT in malaria diagnosis (Cnops et al, 2011 Ishengoma et al. 2011, Veron and Carme, 2006). This confirms PCR on "RDT" as a more reliable tool than microscopy for QC in monitoring the performance of RDT in malaria diagnosis. Equally, in a study by Sandor et al, (2005), PCR on "RDT" showed similar result to PCR on filter paper as source of DNA when chelex method was used for DNA extraction suggesting that the DNA extracted from RDT cassette in this study was pure.

Merozoite surface protein 2 ( $\mathrm{msp} 2$ ) is a protein located on the surface membrane of malaria parasite (Kiwanuka, 2009). Due to the high polymorphic nature of the protein showing length polymorphism, it has been used in several studies to monitor the genetic diversity of malaria parasite (Ghanchi et al, 2010; Farnert and Bjorkman, 2005). As msp 2 was among the genes amplified to detect malaria parasite in this study, we also assessed the genetic diversity of msp 2 from the 37 PCR positive archived RDT samples. From our results, msp 2 showed limited diversity with a low complexity of infection. The alleles ranged from 400-630 bp and the 500bp was the most frequent.

The use of RDT as a source of malaria parasite DNA for molecular analysis by PCR could be of great importance to monitor the test performance of malaria diagnostic tools especially in QC monitoring of RDTs. Also, it could be used as an alternative to filter paper in molecular epidemiologic studies to monitor the genetic diversity, identify drug resistant markers etc. of the parasite. Most molecular epidemiology studies have relied on filter paper as a source of malaria parasite DNA which is costly. Thus, the use of RDT cassettes as a source of DNA will be a more cost effective source of DNA to serve for molecular epidemiologic studies especially in areas were limited studies on malaria are conducted.

\section{Conclusion}

This study confirm that newly used ("fresh") or archived RDT could be a potential source of DNA to detect malaria parasite by PCR and can serve in quality control monitoring of the test performance of RDT. The cost effectiveness of this source of DNA could be of great importance in molecular epidemiologic studies especially in area of declining malaria prevalence where limited studies are been conducted to enhance malaria control and elimination.

\section{References}

Chansuda W, Barcus M.J, Muth S, Sutamihardja A, and Wernsdorfer W.H. (2007). A Review of Malaria Diagnostic Tools: Microscopy and Rapid Diagnostic Test (RDT). American Journal of Tropical Medicine and Hygiene 77: 119-127.

Cnops L, Merel B, Gillet P, Esbroeck M. V, and Jan J. (2011). Rapid diagnostic tests as a source of DNA for Plasmodium species-specific real-time PCR. Malaria Journal 10:67- 75.

Edoh D, Steiger S, Genton B, and Beck H.P. (1997). PCR amplification of DNA from malaria parasites on fixed and stained thick and thin blood films. Transaction of the Royal Society of Tropical Medicine and Hygiene 91:361-363.

Farnert A, and Bjorkman A. (2005). Short report: Limited advantage of multiple consecutive samples for genotyping Plasmodium falciparum populations during the first day of tratment." American Journal of Tropical Medicine and Hygiene 73(1):204-206.

Ghanchi K.N, Andreas M, Johan U, Sana J, Sándor B, Rabia H and Mohammad A. (2010). Genetic diversity among Plasmodium falciparum field isolates in Pakistan measured with PCR genotyping of the merozoite surface protein 1 and 2." Malaria Journal 9:1-6.

Igbinosa O, Igbinosa N, Asowata O, and Chenyi J. (2010) A sequential review on accuracy of detecting 
malaria Parasitemia in developing countries with large restriction on resources." Journal of Medicine and Medical Sciences 1(9): 385-390.

Ishengoma D. S, Lwitiho S, Madebe R.A, Nyagonde N, Ola P, Lasse S.V, Lemnge M.M, and Alifrangis M. (2011). Using rapid diagnostic tests as source of malaria parasite DNA for molecular analyses in the era of declining malaria prevalence." Malaria Journal 10:6-13.

Kiwanuka G. (2009). Genetic diversity in Plasmodium falciparum merozoite surface protein 1 and 2 coding genes and its implications in malaria epidemiology: a review of published studies from 1997-2007." Journal of Vector Borne Diseases 46, 1-12.

Mbacham W. F, Nsangou P. M., Palmer M. N., Evehe B. M-S, Akindeh N, Immaculate A, Johanna D, Valerie M, Kayla L, Songmbe M.Y, Lomah N, Enyong P, and Titanji P.K. (2008). Limited variation of the 5 'cis-control region of the transmission blocking vaccine candidate Pfs 25 amid great genetic diversity of Plasmodium falciparum in Cameroon." African Journal of Biotechnology 7 (5):523-533.

Moody A. (2002). Rapid Diagnostic Tests for Malaria Parasites. Clinical Microbiological reviews 15(2);6678.

NVBCP. (2007). Manual on quality assurance for laboratory diagnosis of Malarial:Rapid Diagnostic Test. National Vector Borne control Programme of the Ministry of Public Health and Ware fare, India 21$33,45-70$.

Plowe C.V, Djimde A, Bouare M, Doumbo O. and Wellems T.E. (1995). Pyrimethamine and proguanil resistance-conferring mutations in Plasmodium falciparum dihydrofolate reductase: polymerase chain reaction methods for surveillance in Africa." American Journal of Tropical Medicine and Hygiene 52: 565-568.

Sandor B, Pedro Gil A M J, and Farnert A. (2005). Rapid DNA extraction from archive blood spots on filter paper for genotyping of Plasmodium falciparum." American Journal of Tropical Medicine and Hygiene 72(3): 249-251

Scopel K.K, Fontes C.J, Nunes A.C, Horta M.F, and Braga E.M. (2004). Low sensitivity of nested PCR using Plasmodium DNA extracted from stained thick blood smears: an epidemiological retrospective study among subjects with low parasitaemia in an endemic area of the Brazilian Amazon region." Malaria Journa/3:8.

Tangpukdee N, Duangdee C, Wilairatana P and Krudsood P. (2009). Malaria Diagnosis: A Brief Review." Korean Journal of Parasitology 47 (2): 93-102.

Veron, V.and Carme B. (2006). Recovery and use of Plasmodium DNA from malaria rapid diagnostic tests. American Journal of Tropical Medicne and Hygiene 74(6) 941-943.

WHO. (2008). Quality Assurance of Malaria Rapid Diagnostic Tests: Buying well andmaintaining accuracy Geneva, Switzerland. World Health Organisation.

WHO. (2010). World malaria report 2010. Global Malaria Programme "WHO/HTM/GMP/2010.1". http://www.who.int/malaria.

\section{Tables}

Table 1: Amplification success of DNA extracted from archive RDT

\begin{tabular}{|c|c|c|c|}
\hline \multirow[t]{2}{*}{ RDT sample category } & \multirow[t]{2}{*}{ Number of RDT } & \multicolumn{2}{|c|}{ PCR } \\
\hline & & Positive (\%) & Negative (\%) \\
\hline & Fresh RDT & & \\
\hline \multirow[t]{2}{*}{ RDT positive } & 4 & $4(100 \%)$ & $0(0 \%)$ \\
\hline & Archive RDT & & \\
\hline RDT and microscopy positive & 12 & $12(100 \%)$ & $0(0 \%)$ \\
\hline RDT negative but microscopy positive & 14 & $14(100 \%)$ & $0(0 \%)$ \\
\hline RDT positive but microscopy negative & 11 & $11(100 \%)$ & $0(0 \%)$ \\
\hline RDT and microscopy negative & 6 & $0(0 \%)$ & $6(100 \%)$ \\
\hline Total & 47 & 41 & 6 \\
\hline
\end{tabular}


Table 2: Allelic distribution and complexity of infection for Plasmodium falciparum

\begin{tabular}{ccccccc}
\hline $\begin{array}{c}\text { Number of } \\
\text { samples }\end{array}$ & $\begin{array}{c}\text { Number of DNA } \\
\text { fragments }\end{array}$ & $\begin{array}{c}\text { Number of } \\
\text { alleles }\end{array}$ & $\begin{array}{c}\text { Genetic } \\
\text { diversity (\%) }\end{array}$ & $\begin{array}{c}\text { Complexity of } \\
\text { infection }\end{array}$ & $\begin{array}{c}\text { Mean } \\
\text { allelic size } \\
\text { (bp) }\end{array}$ & $\begin{array}{c}\text { Range of msp } \\
\text { 2 alleles (bp) }\end{array}$ \\
\hline $\mathbf{3 7}$ & 48 & 17 & $35.4 \%$ & 1.3 & $528.15 \pm 57$ & $400-620$ \\
\hline
\end{tabular}

Legend: bp; base pair

\section{Figures}

A
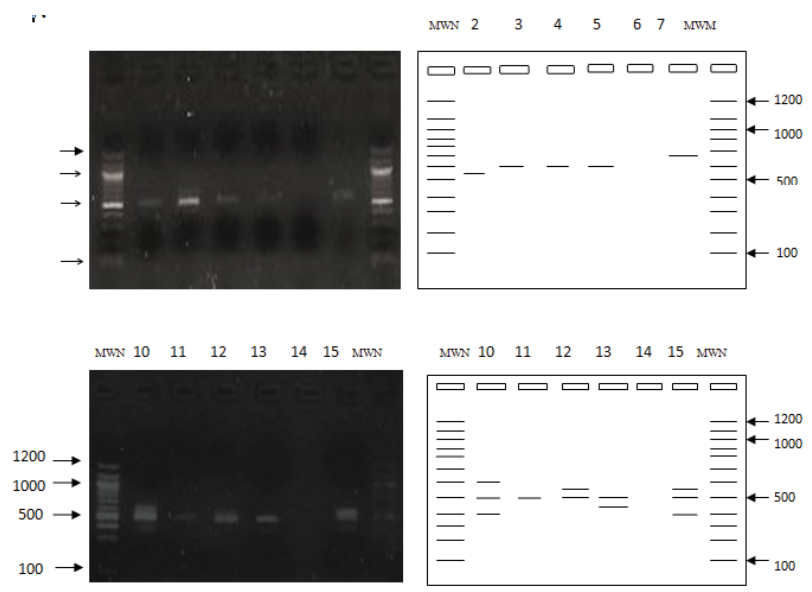

B

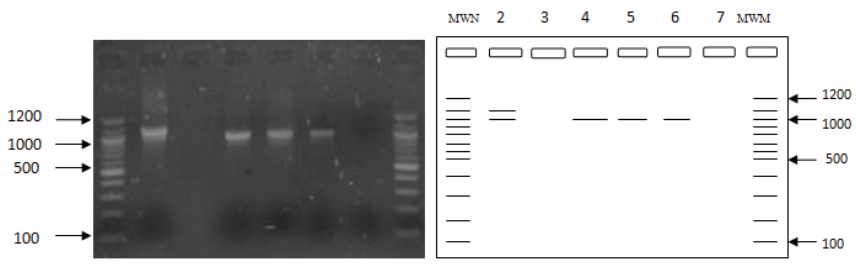

C

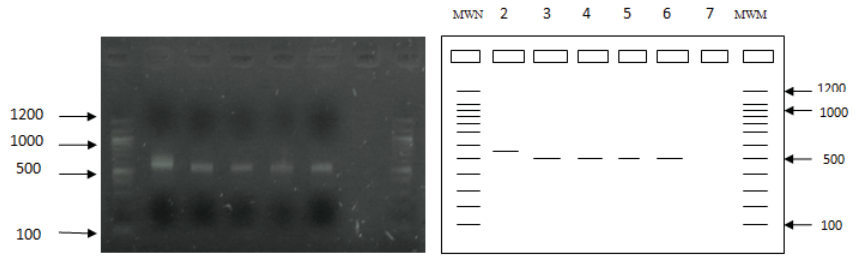

Figure 1: Agarose gel electrophoresis of nested PCR product

Legend: Lane MWM: Molecular weight marker ( in base pairs), 3A: Positive result for msp 2; lane 2-5 and 10-13, Negative result for msp 2; lane 6, Positive control; lane 7 and 15, 3B: Positive result for glurp (4 RDT replicates); lane 4, 5 and 6, lane 2; positive control, lane 7; Negative result for glurp. 3C: Positive samples for msp 1 (4 RDT replicates); lane 2, 4, 5 and 6, lane 1; positive control. 


\section{P. falciparum msp 2 infection}

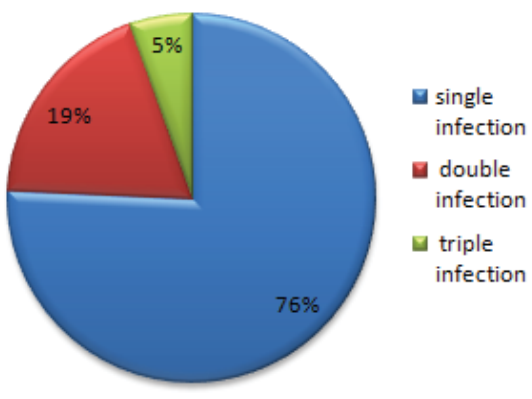

Figure 2: Distribution of Plasmodium falciparum msp 2 infections

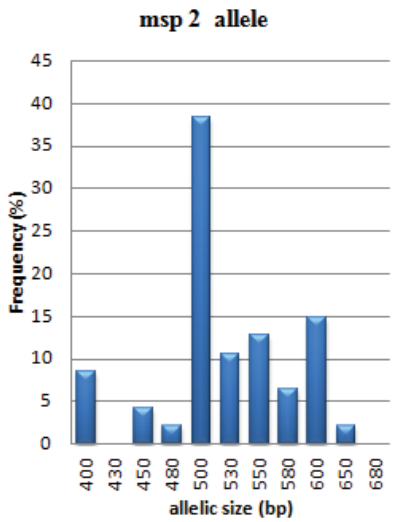

Figure 3: Allelic distribution of Plasmodium falciparum msp 2 gene 
